\title{
Sorting radar signal from symmetry clustering perspective
}

\author{
Mohaned Giess Shokrallah Ahmed* and Bin Tang \\ School of Electronic Engineering, University of Electronic Science and Technology of China, Chengdu 611731, China
}

\begin{abstract}
The main function of electronic support measure system is to detect threating signals in order to take countermeasures against them. To accomplish this objective, a process of associating each interleaved pulse with its emitter must be done. This process is termed sorting or de-interleaving. A novel point symmetry based radar sorting (PSBRS) algorithm is addressed. In order to deal with all kinds of radar signals, the symmetry measure distance is used to cluster pulses instead of the conventional Euclidean distance. The reference points of the symmetrical clusters are initialized by the alternative fuzzy c-means (AFCM) algorithm to ameliorate the effects of noise and the false sorting. Besides, the density filtering (DF) algorithm is proposed to discard the noise pulses or clutter. The performance of the algorithm is evaluated under the effects of noise and missing pulses. It has been observed that the PSBRS algorithm can cope with a large number of noise pulses and it is completely independent of missing pulses. Finally, PSBRS is compared with some benchmark algorithms, and the simulation results reveal the feasibility and efficiency of the algorithm.
\end{abstract}

Keywords: sorting radar pulse, symmetry, alternative fuzzy c-means, noise, missing pulse.

DOI: $10.21629 /$ JSEE.2017.04.08

\section{Introduction}

Electronic support measure (ESM) is one of the essential parts of electronic warfare (EW), which is mainly used for intercepting, measuring, and sorting hostile signals [1]. Since all emitters transmit their signals through one channel, the ESM receiver intercepts the radar pulses in an interleaved form, and it is very difficult to discriminate between these signals. The process of associating each pulse with its radar emitter is termed "sorting" or "deinterleaving" $L$, and it is absolutely vital for taking countermeasure against hostile signals.

Extensive algorithms have been proposed in order to tackle this issue [2-4]. They achieve good performance for small numbers of radar signals. However, their

\footnotetext{
Manuscript received May 25, 2016.

*Corresponding author.

This work was supported by the National Natural Science Foundation of China (61172116).
}

performances are rapidly deteriorated in the presence of missing pulses and staggered signals. In [5], a new validity index (VIX) was proposed to enhance the sorting results. Although VIX overcomes the effects of missing pulses and jittered noise, the authors did not mention how to suppress these noise pulses from the radar signals. Besides, the VIX has to be adopted many times to obtain the optimal number of clusters, therefore, the computation burden is high. In [6], a combined algorithm was proposed to ameliorate the sorting performances in high pulse rate density. The algorithm assumed that the ESM's receiver can intercept a prohibitive number of pulses for each emitter, which is practically unrealistic. An improved dynamic density clustering (IDDC) was investigated in [7] for improving the efficiency of the sorting. IDDC demonstrated high performances for staggered and jittered signals. However, it is very sensitive to some tolerance vectors, which must be specified by the user. Another weakness in the IDDC algorithm is that, it can be only deployed for pulses in the form of hyper-spherical clusters.

In an effort to mitigate the deficiencies of the previous conventional algorithms, a point symmetry based radar sorting (PSBRS) algorithm is proposed in this paper. Unlike most sorting algorithms, PSBRS does not implement Euclidean distance to measure the dissimilarity between pulses. It deploys the so-called 'point symmetry' technique [8-11]. Symmetry is a common feature in the world, and it is rationale to assume that this feature is exited in the clusters. The idea behind the point symmetry measure is to detect the assumed symmetry from the clusters. It associates the pulses with a cluster centroid if they are symmetrical. Adopting symmetry distance instead of Euclidean distance gives the investigated algorithm an advantage over the existing sorting algorithms, and that is capable of detecting hyper-ellipsoidal, hyper-spherical and any other clusters' shapes.

It is worth notice that initial reference points are sensitive to the performance of the symmetrical clustering. In [9], the well-known k-means algorithm was employed to 
obtain the initial reference points of the symmetrical clusters. Nevertheless, this work reveals that the k-means algorithm cannot assure efficient clustering if its resultant centers are lain on the middle between two close clusters. Additionally, the k-means algorithm is likely to provide empty clusters, hence, the number of initial centroids is less than the number of clusters. In this paper, an alternative fuzzy cmeans (AFCM) algorithm is considered to ameliorate the initial reference points of symmetrical clusters. One major advantage of AFCM is that the resultant centers are not strongly influenced by the noise or the close clusters, therefore, the centroids will not be located at the intermediate distance between symmetrical clusters.

Generally, noise pulses produce further disturbances in the final sorting results, and it should be removed. To this end, a density filtering (DF) algorithm is considered to discard the noise pulses.

The contributions of this paper can be summarized as follows. (i) The capability of PSBRS to sort modern radar signals, such as staggered, frequency hopping, and jittered signals. (ii) The outstanding performance of PSBRS under the influence of noise and missing pulses. (iii) Proposing a symmetry clustering algorithm, which to the best of our knowledge, is used for the first time for sorting radar signals.

The residual of this paper is systemized as follows. Section 2 gives preamble to the distance metrics, while Section 3 demonstrates the proposed method. The simulation results are provided in Section 4, and finally this paper is concluded in Section 5.

\section{Preamble to the distance metrics}

\subsection{Euclidean distance}

To sort radar pulses by using the clustering techniques, the distance measure between pulses must be computed. The well-known distance measure is Euclidean distance, which obtains the dissimilarities between objects. Let $U=$ $\left.u_{i}\right]_{i=1}^{N}$ represent the received pulses in $v$ dimension space, i.e. $\bar{U} \subseteq R^{v}$. The Euclidean distance, $d_{E d}$, between the $m$ th and $n$th pulses is given as follows:

$$
d_{E d}(m, n)=\left(\sum_{V=1}^{v}\left|u_{m V}-u_{n V}\right|^{2}\right)^{1 / 2} .
$$

Euclidean distance is commonly used in clustering and data mining. Despite its popularity, it can only deal with hyper-spherical clusters. Generally, radar pulses may take different shapes based on the type of signals (staggered, agility, frequency hopping, etc.). Since there is no priorknowledge about the intercepted signal, Euclidean distance becomes an inefficient measure for clustering radar pulses.

\subsection{Symmetry measure}

Symmetry is the most common feature in the world, and most objects around us consist of a qualitative and generalized form of symmetry. Therefore, it can be assumed that the structures of clusters have some kinds of symmetry. It is based on the idea that pulses can be associated with a center if they give a symmetrical structure with respect to the center. The point symmetry distance can be obtained as follows. Given the $N$ received pulses and a reference point $c$, the point symmetry distance between the data point $u_{j}$ and a reference point $c$ [9] can be defined as

$$
d_{p s}\left(u_{j}, c\right)=\min _{\substack{j=1, \ldots, N \\ j \neq i}} \frac{\left\|\left(u_{j}-c\right)+\left(u_{i}-c\right)\right\|}{\left(\left\|u_{j}-c\right\|+\left\|u_{i}-c\right\|\right)}
$$

where $\left(\left\|u_{j}-c\right\|+\left\|u_{i}-c\right\|\right)$ is a normalization term. If (2) is minimized when $u_{i}=u_{j^{*}}$, the pulse $u_{j^{*}}$ will be the symmetrical pulse relative to $u_{j}$ with respect to $c$. Notice that the minimization can be equal to zero if and only if $u_{j^{*}}=2 c-u_{j}$, i.e. $d_{p s}\left(u_{j}, c\right)=0$.

In order to clarify the concept of symmetry, four pulses are delineated in Fig. 1, i.e. $\boldsymbol{U}=\left[u_{1}, u_{2}, u_{3}, u_{4}\right]$, and one reference point $c$. The main objective is to obtain the symmetrical pulses with respect to the reference point $c$.

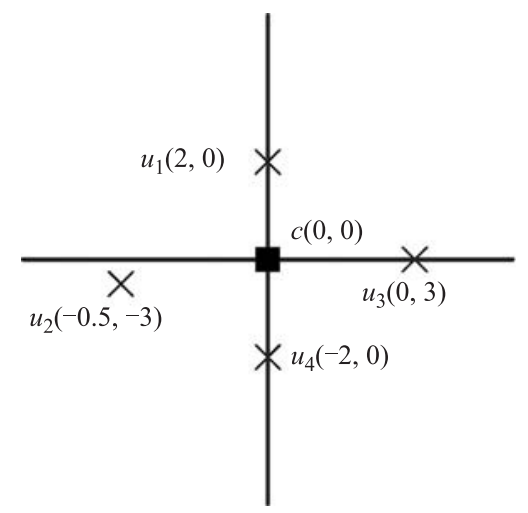

Fig. 1 Symmetrical pulses with respect to the reference point $c$

The minimum point symmetry distances are calculated as follows:

$$
\begin{gathered}
d_{p s}\left(u_{1}, c\right)=\frac{\left\|\left(u_{1}-c\right)+\left(u_{2}-c\right)\right\|}{\left(\left\|u_{1}-c\right\|+\left\|u_{2}-c\right\|\right)}=\frac{0.5}{\sqrt{9.25}}=0.164 \\
d_{p s}\left(u_{2}, c\right)=\frac{\left\|\left(u_{2}-c\right)+\left(u_{1}-c\right)\right\|}{\left(\left\|u_{2}-c\right\|+\left\|u_{1}-c\right\|\right)}=\frac{0.5}{\sqrt{9.25}}=0.164 \\
d_{p s}\left(u_{3}, c\right)=\frac{\left\|\left(u_{3}-c\right)+\left(u_{4}-c\right)\right\|}{\left(\left\|u_{3}-c\right\|+\left\|u_{4}-c\right\|\right)}=\frac{0}{4}=0 \\
d_{p s}\left(u_{4}, c\right)=\frac{\left\|\left(u_{4}-c\right)+\left(u_{3}-c\right)\right\|}{\left(\left\|u_{4}-c\right\|+\left\|u_{3}-c\right\|\right)}=\frac{0}{4}=0 .
\end{gathered}
$$

It is apparent that the pulses $\left(u_{1}, u_{2}\right)$ and $\left(u_{3}, u_{4}\right)$ are symmetrical. In other words, pulses $u_{1}$ and $u_{2}$ are associated 
with one cluster, while pulses $u_{3}$ and $u_{4}$ are associated with another cluster. The next question is: how can we obtain the best reference points?

$\mathrm{K}$-means is one of the common partition clustering algorithms, and it uses Euclidean distance to measure the dissimilarity between objects [8]. One major weakness of the $\mathrm{k}$-means algorithm is that, it might not converge into the global solution.

The authors in [9] incorporated k-means into symmetry clustering for enhancing the performance of the k-means algorithm. The main idea was that, resultant centers of $\mathrm{k}$ means are considered as initial reference points, and then the symmetry clustering is implemented.

The performance of symmetry measure is enhanced by considering the Euclidean distance and the nearest two points from the symmetrical points $[10,11]$. Thus, the improved symmetry measure will be

$$
d_{p s}\left(u_{j}, c\right)=\frac{\left(d_{1}+d_{2}\right)}{2} \times d_{E d}\left(u_{j}, c\right)
$$

where $d_{1}$ and $d_{2}$ are the first and second nearest pulses from the symmetrical point $u_{j}^{*}$, respectively.

\section{The proposed methods}

\subsection{Initial reference points}

Fig. 2 shows three clusters and three reference points. All the reference points are initialized by the conventional kmeans. The two spherical clusters $C l_{1}$ and $C l_{2}$ are close together, and the reference point $c_{3}$ is located between them. The third cluster $\mathrm{Cl}_{3}$ has elongated shape, and the reference points $c_{1}$ and $c_{2}$ are located inside it. Suppose $u_{m}$ is an examinable pulse, then the point symmetry distance from the pulse $u_{m}$ to each reference point will be computed as follows.

$$
\begin{aligned}
& d_{p s}\left(u_{m}, c_{1}\right)=\frac{d_{E d 11}+d_{E d 21}}{2} \times d_{E d}\left(u_{m}, c_{1}\right) \\
& d_{p s}\left(u_{m}, c_{2}\right)=\frac{d_{E d 12}+d_{E d 22}}{2} \times d_{E d}\left(u_{m}, c_{2}\right) \\
& d_{p s}\left(u_{m}, c_{3}\right)=\frac{d_{E d 13}+d_{E d 23}}{2} \times d_{E d}\left(u_{m}, c_{3}\right)
\end{aligned}
$$

where $d_{E d 1 i}$ and $d_{E d 2 i}$ represent the first and second nearest Euclidean distance from the symmetrical point $u_{m i}$, respectively. Assume that $c_{3}$ is the nearest reference point to the examinable pulse $u_{m}$, while $c_{1}$ and $c_{2}$ are apart from it. In this instance, $d_{p s}\left(u_{m}, c_{3}\right)$ is the smallest symmetry distance, and $d_{E d 13}$ and $d_{E d 23}$ are the nearest distance from the symmetrical point $u_{m 3^{*}}$. If $c_{3}$ is in the middle between the two close clusters, then each pulse in $C l_{1}$ will find its symmetrical point in $\mathrm{Cl}_{2}$. Therefore, $\mathrm{Cl}_{1}$ and $\mathrm{Cl}_{2}$ will be clustered as a unique cluster, while $\mathrm{Cl}_{3}$ will be divided into two clusters.

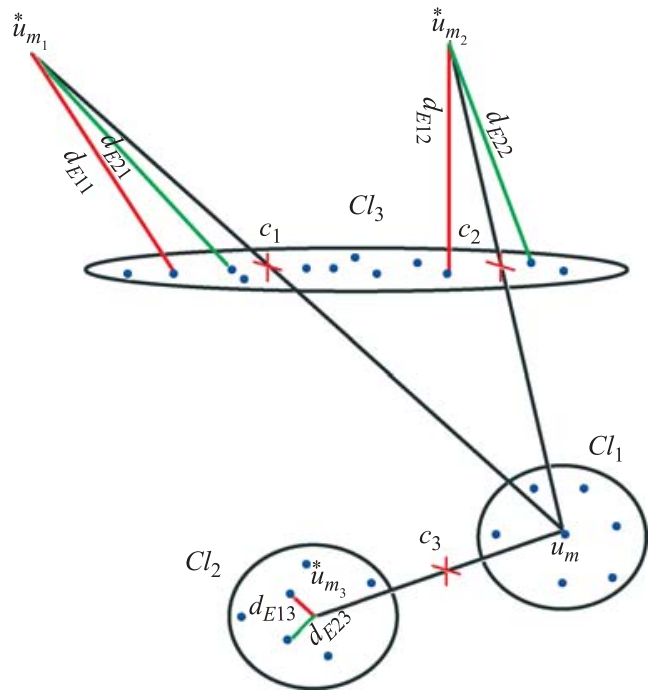

Fig. 2 Case where the symmetrical algorithm cannot recognize the correct clusters

Obviously, the symmetrical clustering will lead to wrong results for the previous case. In order to guarantee correct clustering, the initial reference points must not be in the middle between clusters. The objective now is to find an appropriate method for initializing the reference points.

\subsection{AFCM algorithm}

Fuzzy c-means (FCM) is a generalization of k-means, and it performs better than the k-means algorithm. However, it has two considerable difficulties:

(i) Sensitive to noise pulses.

(ii) Sensitive to different cluster shapes.

The AFCM algorithm was proposed by Wu and Wang to overcome these weaknesses [12]. The idea of AFCM is similar to FCM except that it relies on a new metric that is non-sensitive to noise pulses and clusters' shapes.

Let the received radar pulses sequence be $\left.U=u_{i}\right]_{i=1}^{N}$, the best estimate of the center can be obtained by minimizing the mean square error with respect to $c$ in [12] as

$$
\sum_{j=1}^{N}\left\|u_{j}-c\right\|^{2} .
$$

Then,

$$
c=\frac{\sum_{j=1}^{N} u_{j}}{N} .
$$

Equation (8) can be rewritten as follows.

$$
c=\sum_{j=1}^{N} \frac{w_{j}}{\sum_{j=1}^{N} w_{j}} u_{j}
$$


where $w_{j}$ is the weight of the $j$ th pulse. Equations (8) and (9) are equivalence if $w_{j}=1$. Though, if we manipulate these weights, such that, noisy pulses have small weights, while radar pulses have large weight, then the resultant centers will be more robust. In an effort to achieve this goal, the alternative metric will take the following form.

$$
d(u, c)=1-\exp \left(-\gamma\|u-c\|^{2}\right)
$$

where $\gamma$ is constant, and it is defined by

$$
\gamma=\left(\frac{\sum_{j=1}^{N}\left\|u_{j}-c\right\|^{2}}{N}\right)^{-1} .
$$

Based on (10) the AFCM cost function is defined as

$$
J_{A F C M}=\sum_{r=1}^{k} \sum_{j=1}^{N} \mu_{i r}^{q} \times\left(1-\eta_{j r}\right)
$$

where $q>1, \sum_{i=1}^{c} \mu_{i j}=1$, and $\eta_{j r}=\exp \left(-\gamma\left\|u_{j}-c_{r}\right\|^{2}\right)$. The objective function in (12) will be minimized if

$$
\mu_{j r}=\frac{\left[1 /\left(1-\eta_{j r}\right)\right]^{1 / q-1}}{\sum_{r=1}^{k}\left[1 /\left(1-\eta_{j r}\right)\right]^{1 / q-1}}
$$

and

$$
c_{r}=\frac{\sum_{j=1}^{N} \mu_{j r}^{q} \eta_{j r} u_{j}}{\sum_{j=1}^{N} \mu_{j r}^{q} \eta_{j r}} .
$$

The steps of AFCM can be summarized as follows.

Step 1 Set the iteration counter $w=0$.

Step 2 Initialize the centers randomly $c_{r}^{w}, r=1, \ldots, k$.

Step 3 Obtain the membership values $\mu_{j r}^{w}$ from (13).

Step 4 Update the centers from (14), where the fuzzivariable $q>1$.

Step 5 If $\left\|c_{r}^{w+1}-c_{r}^{w}\right\|<\varepsilon$, then stop. Otherwise, $w=w+1$ and go to Step 3 .

Implementing AFCM instead of k-means performs better estimation of reference points. Also, we can guarantee that the centers will not be located between the clusters, and they will move toward clusters based on the constant value of $\gamma$.

In order to compute the time complexity of AFCM, assume that the constant $\gamma$ is stored in the memory and computed once for all iterations. Thus it does not affect the time complexity. The term $\mu_{j r}$ is calculated once per data pulse, and cost $O(N)$ for each center, leading to a cost $O(N k)$ per iteration. For the centers $c_{r}$, since the matrix $\boldsymbol{\eta}_{j r}$ is already computed when the membership $\mu_{j r}$ is obtained, then we just need to compute it for each center, hence, it costs $O(k)$ per iteration. If the total number of iterations is $\tau$, then the time complexity of the $\mathrm{KFCM}$ is $O(\tau(N k+k))$.

\subsection{DF algorithm}

The noise pulses or clutter must be discarded before deploying the symmetrical clustering. Without discarding the noise pulse, it is impossible to differentiate between noise pulses and radar pulses. Let $\left.U=u_{j}\right]_{j=1}^{N}$ represent the received signal with some noise pulses. From scientific point of view, noise pulses can be recognized by its scattering feature, that is, any pulse which does not have neighborhoods within a specific radius will be considered as noise pulse. Thus, the DF algorithm can be obtained by the following steps.

Step $1 j=1$.

Step 2 Calculate the Euclidean distance $d_{E d}$ between $u_{j}$ and all remaining pulses.

Step 3 If the minimum $d_{E d}$ is less than some threshold $T h$, then $u_{j}$ is a radar pulse. Otherwise $u_{j}$ is a noise pulse.

Step $4 j=j+1$, until all pulses are examined.

The choice of the threshold is absolutely critical, and it can be obtained by experiences. If $T h$ is larger than required, then no noise pulse will be detected. Else if $T h$ is smaller than required, all pulses will be detected as noise.

Note that the Euclidean distance is computed between each pulse and the remaining pulses, therefore, it costs $O(N-1)$ for each pulse, leading to $O(N(N-1))$ for all pulses.

Therefore, adopting AFCM and DF algorithms on symmetry clustering leads to $O(\tau(N k+k)+(N(N-1)))$ in total.

\subsection{PSBRS steps}

For simplicity, the steps of the PSBRS algorithm can be summarized as follows.

Step 1 Initialization: Normalize the parameters; apply the DF algorithm, and then randomly choose $k$ cluster centroids from data points.

Step 2 Rough-tuning: Apply the AFCM algorithm to update the initial centroids until the cluster centroids are converged or some stopping criteria are satisfied. These centers are considered as reference points.

Step 3 Precise-tuning: Obtain the associated reference point with each pulse based on

$$
\widetilde{r}=\arg \min _{r=1, \ldots, k} d_{p s}\left(u_{j}, c_{r}\right)
$$


where $d_{p s}\left(u_{j}, c_{r}\right)$ is defined by (3). If the symmetry distance is less than some threshold $\xi$, then the pulse $u_{j}$ will be assigned to the $\widetilde{r}$ th cluster. Else, the pulse $u_{j}$ will be assigned to the $\widetilde{r}$ th cluster based on

$$
\widetilde{r}=\arg \min _{r=1, \ldots, k} d_{E d}\left(u_{j}, c_{r}\right) .
$$

Step 4 Updating stage: Update the reference points as follows.

$$
c_{r}=\frac{\sum_{i \in C l_{r}} u_{i}}{N_{r}}
$$

where $C l_{r}$ represents all the associated data points with the $r$ th cluster, while $N_{r}$ is the number of points in the $r$ th cluster.

Step 5 Termination stage: The algorithm will be terminated if the data pulses in each cluster are remained constant. Else, go to the second step.

\section{Simulation results}

In order to investigate the efficiency of the PSBRS algorithm, six radar signals are simulated as in Table 1.

Table 1 The feature of the simulated radar

\begin{tabular}{cccccc}
\hline$R$ & PRI/ms & PW/ $/ \mathrm{s}$ & RF/MHz & DOA & Number \\
\hline 1 & $0.52-0.58$ & $1.15-1.25$ & $2400-2550$ agility & $28-40$ & 130 \\
2 & $0.8-1$ & $1.35-1.40$ & $2000-2150$ hopping & $48-60$ & 120 \\
3 & $0.76-0.84$ & $1.25-1.35$ & $2855-3.050$ agility & $15-25$ & 125 \\
4 & $0.5-0.6$ & $0.95-1.05$ & $3050-3.15$ agility & $40-60$ & 130 \\
5 & $0.6-0.62$ & $1.04-1.12$ & $1500-1600$ hopping & $30-40$ & 115 \\
6 & $0.42-0.46$ & $1.27-1.34$ & $5200-5300$ agility & $30-50$ & 130 \\
\hline
\end{tabular}

Firstly, the conventional k-means and the AFCM are implemented in the received signal as delineated in Fig. 3(a) and Fig. 3(b), respectively. Evidently, one resultant center of k-means is laid between the two symetrical clusters, while all centers are moved towards clusters when AFCM is implemented.

Fig. 3(c) shows the symmetry clustering results after using the k-means centers as initial reference points. Even though the symmetry clustering is implemented, it cannot differentiate between the two red clusters, and the centroid is still located between them, simply because every pulse in the first red cluster has found its symmetrical pulse in the other cluster. In contrast, Fig. 3(d) depicts the symmetry clustering based on the resultant AFCM centers. It is clear that the symmetry algorithm detects all the clusters well.

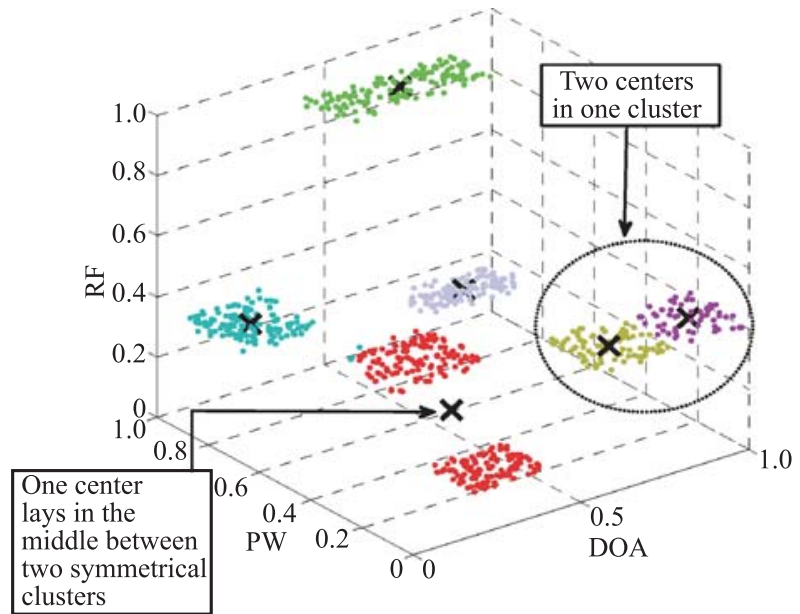

(a) Standard k-means results

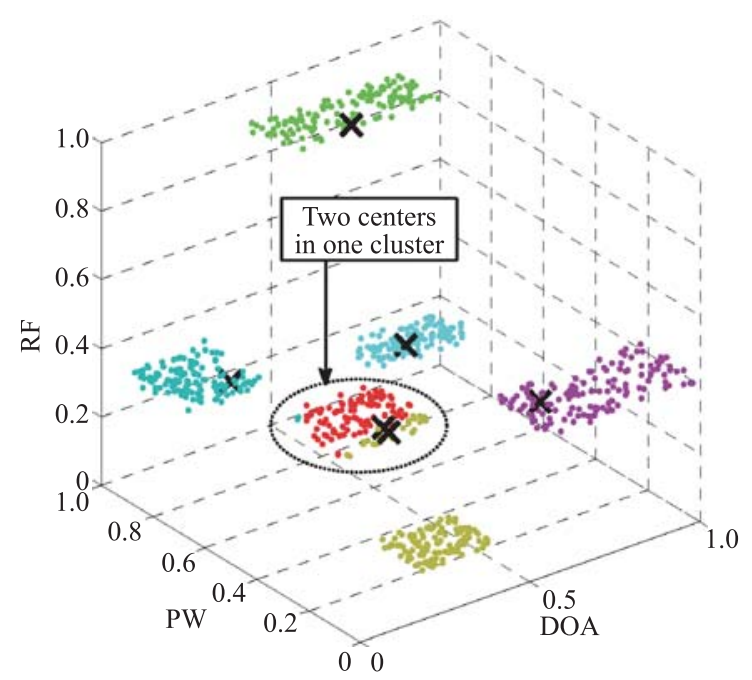

(b) AFCM clustering result

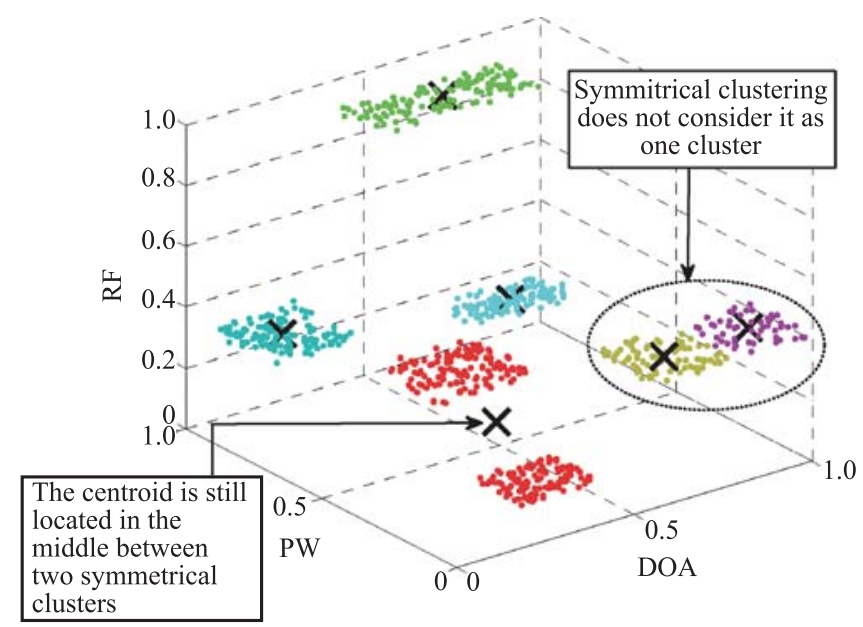

(c) Symmetry clustering with k-means 


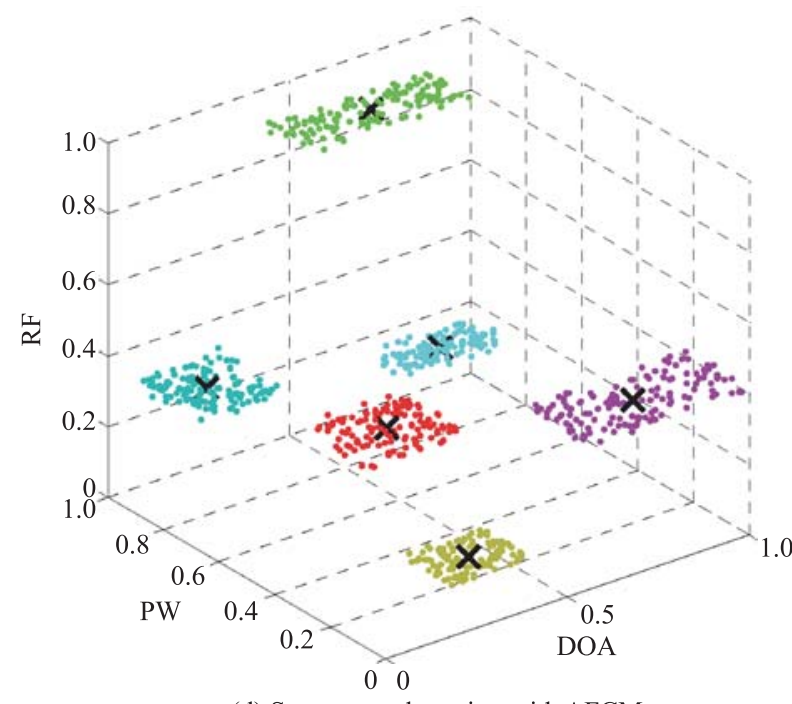

(d) Symmetry clustering with AFCM

Fig. 3 Clustering results

Secondly, the influence of noise pulses is examined and compared with some benchmark sorting algorithms, i.e. IDDC [7], sorting radar algorithm based density (SRBD) technique [13], and FCM [5]. Fig. 4 demonstrates the average root mean square error (ARMSE) [14] versus the number of noise pulses. The Monte Carlo simulations are conducted 300 times, and the ARMSE is calculated as follows.

$$
\text { ARMSE }=\frac{\sum_{r=2}^{k} \sqrt{\frac{\sum_{j=1}^{N}\left(A c c_{j}-\overline{A c c}\right)^{2}}{N}}}{k-1}
$$

where $A c c_{j}$ denotes the accuracy of the $j$ th pulse, and it ranges between 0 and $1 ; \overline{A c c}$ represents the maximum accuracy, and it is equal to one; $c$ denotes the number of radars.

From Fig. 4, it is apparent that the RSBRS algorithm can cope perfectly with 30 noise pulses, while the ARMSE is slightly deteriorated for more than 30 noise pulses. This result is reasonable, because the DF algorithm discards the noise pulses based on their density. When the number of noise pulses is small, the noise pulses are quite separated, therefore, all noise pulses will be detected, while when the number of pulses is large, some noise pulses are gathered together, and then the DF cannot discriminate between them. In contrast, the SRBD algorithm is independent of noise, and its ARMSE is fluctuated around 0.03. Similarly, the FCM algorithm is independent of noise, and its ARMSE is less than PSBRS. IDDC has the worst performances, and it is increased proportional to the number of noise.

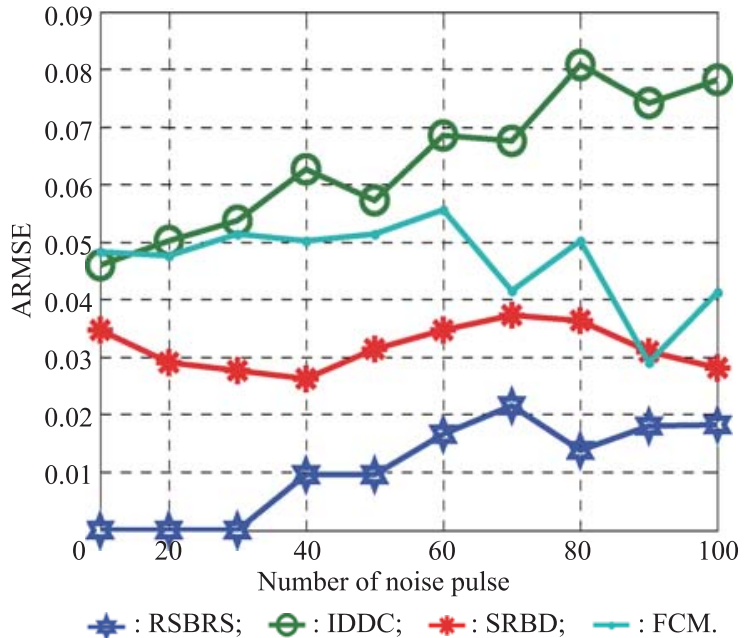

Fig. 4 ARMSE versus noise pulses over 300 Monte Carlo simulations

Fig. 5 illustrates the ARMSE versus the missing pulses percentage. The ARMSE is also from 300 Mote Carlo simulations using (18). All the algorithms are almost independent of missing pulses. It is obvious that PSBRS has the superlative performances, and it is not affected by missing pulses.

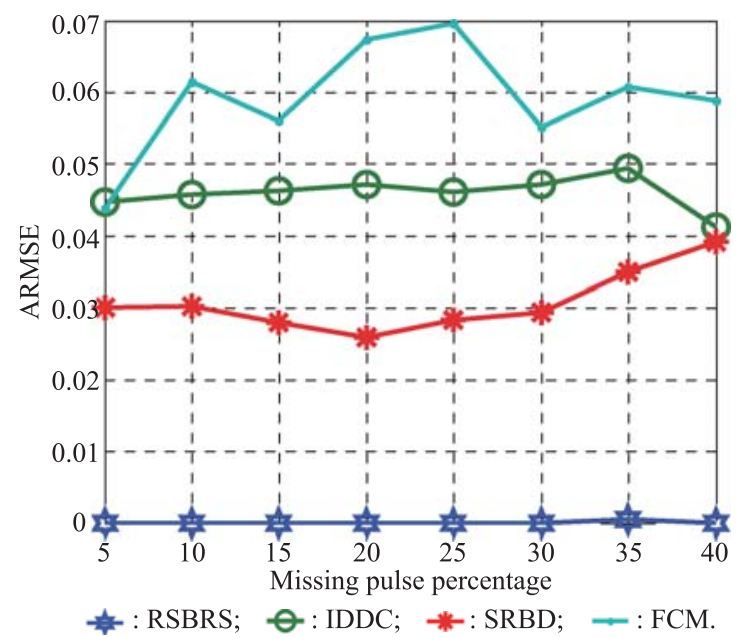

Fig. 5 ARMSE versus missing pulses over 300 Monte Carlo iterations

\section{Conclusions}

In this work, a PSBRS algorithm is investigated for sorting radar signals. Unlike conventional symmetry clustering algorithms, PSBRS obtains the initial reference points based on AFCM, mainly because AFCM overcomes the influence of noise pulses and bad convergence. One advantage of deploying symmetry clustering instead of the conventional sorting algorithms is that, it can detect all kinds of clusters. The DF algorithm is proposed and incorporated into PSBRS to get rid of noise pulses. The performances 
of PSBRS are evaluated under the influence of noise and missing pulses. We have found that the ARMSE is very low for large number of rand noise pulses, and the proposed algorithm is independent of missing pulses. The main weakness of PSBRS is that, the number of radars must be known previously, and the future work may focus on this issue.

\section{References}

[1] R. G. Wiley. ELINT: The interception and analysis of radar signals. London: Artech House on Demand, 2006.

[2] D. J. Milojević, B. M. Popović. Improved algorithm for the deinterleaving of radar pulses. Radar \& Signal Processing IEE Proceedings F, 1992, 139(1): $98-104$.

[3] H. K. Mardia. New techniques for the deinterleaving of repetitive sequences. Radar \& Signal Processing IEE Proceedings $F, 1989,136(4): 149-154$.

[4] K. I. Nishiguchi, K. Masaaki. Improved algorithm for estimating pulse repetition intervals. IEEE Trans. on Aerospace and Electronic Systems, 2000, 36(2): 407-421.

[5] M. G. S. Ahmed, B. Tang. New FCM's validity index for sorting radar signal. Proc. of the IEEE 17th International Conference on Computational Science and Engineering, 2014: 127 131.

[6] Q. Guo, X. Zhang, Z. Li. SVC \& k-means and type-entropy based de-interleaving/recognition system of radar pulses. Proc. of the IEEE International Conference on Information Acquisition, 2006: $742-747$.

[7] A. L. He, D. G. Zeng, W. Jun, et al. Multi-parameter signal sorting algorithm based on dynamic distance clustering. Journal of Electronic Science And Technology of China, 2009, 7(3): $249-253$.

[8] K. Jain. Data clustering: 50 years beyond K-means. Pattern Recognition Letters, 2008, 31(8): 651-666.

[9] S. Chun, C. Chou. A modified version of the K-means algorithm with a distance based on cluster symmetry. IEEE Trans. on Pattern Analysis \& Machine Intelligence, 2001, 23(6): $674-680$.
[10] C. H. Chou, M. C. Su, E. Lai. Symmetry as a new measure for cluster validity. Proc. of the Wseas International Conference on Scientific Compuation \& Soft Computing, 2002: 209-213.

[11] S. Bandyopadhyay, S. Saha. GAPS: A clustering method using a new point symmetry-based distance measure. Pattern Recognition, 2007, 40(12): 3430-3451.

[12] K. Wu, M. Yang. Alternative c-means clustering algorithms. Pattern recognition, 2002, 35(10): $2267-2278$.

[13] X. Wang, X. Zhang, R. Tian, et al. A new method of unknown radar signals sorting. Electronic Information Warfare Technology, 2012, 256: 727-733.

[14] A. Liu, G. Liao, L. Ma, et al. An array error estimation method for constellation SAR systems. IEEE Geosience and Remote Sensing Letters, 2010, 7(4): 731 - 735.

\section{Biographies}

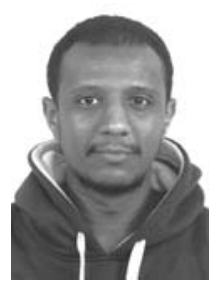

Mohaned Giess Shokrallah Ahmed was born in 1987. He received his B.S. degree in electrical engineering from Karary University in 2009, Khartoum Sudan and M.S. degree in electronic engineering, information and signal processing from University of Electronic Science and Technology of China in 2013, Chengdu, China. He is currently working toward his Ph.D. degree in electronic engineering from University of Electronic Science and Technology of China. His current research interests include radar countermeasure and radar signal processing.

E-mail: 2258042252@qq.com

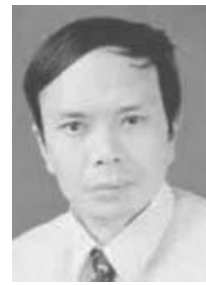

Bin Tang was born in 1964. He received his Ph.D. degree from University of Electronic Science and Technology of China (UESTC). He is currently a professor in the School of Electronic Engineering, UESTC. His research interests include electronic scout and countermeasure, digital signal processing, and wireless communications.

E-mail: bint@uestc.edu.cn 